\title{
Model of interacting dark energy in five-dimensional brane cosmology
}

\author{
Mubasher Jamil* \\ Center for Advanced Mathematics and Physics \\ National University of Sciences and Technology \\ EEMME campus, Peshawar road, Rawalpindi - 46000, Pakistan
}

November 3, 2018

\begin{abstract}
We here investigate the model of interacting dark energy in the context of five dimensional brane cosmology. The effective equations of state of dark energy are evaluated for various choices of the variable time dependent cosmological constant. We have found that the interacting dark energy obeys the phantom divide/crossing scenario in this generalized model. It is also shown that interacting dark energy in this generalized model also resolves the cosmic coincidence problem.
\end{abstract}

Keywords: Dark energy; Chaplygin gas; cosmic coincidence problem; cosmological constant; dark matter; phantom energy; phantom crossing.

\section{Introduction}

Recent astrophysical observations give bunch of convincing evidence of our universe undergoing accelerated expansion [1, 2, 3, 4, 5, 6, 7, 8]. A perturbing feature of this phenomenon is that it was preceded by a decelerated expansion, so we need to ask what caused this sudden transition [9]. Observations also show that this shift is rather marginally recent (less then one Gyr), hence it poses why it happened so recently. If we assume that the source which is driving this expansion is some mysterious 'dark energy' then one needs to ask what is the composition of this exotic matter, also why it has become dominating all

*Email: mjamil@camp.edu.pk 
of a sudden at present time (see [10, 11, 12, 13, 14] for reviews on dark energy). In other words, why it was negligibly small in earlier deceleration phase. Investigations of WMAP show that energy densities of dark energy and matter are almost comparable at present time. This leads to a problem named as the 'cosmic coincidence problem' (CCP). The problem aggravated when it was shown that radiation energy density was also equivalent to that of dark energy, leading to a 'cosmic triple coincidence problem' [15], however it is recently addressed in the context of triple interacting fluids [16]. Further if the universe is dark energy dominated, will it expand forever or may decelerate at some instant as well.

The dark energy is generally represented by a phenomenologically motivated equation of state $(\mathrm{EoS}) p_{d e}=\omega_{d e} \rho_{d e}$, where $p_{d e}$ and $\rho_{d e}$ are the pressure and the energy density of the dark energy, interlinked by a dimensionless parameter $\omega_{d e}$ [17]. In order to produce the accelerated expansion, it requires $\omega_{d e}<-1 / 3$. In recent years, several theoretical models have been proposed to understand the nature and dynamics of dark energy, however almost all these models either require fine tuning of their model parameters or yield quantum or gravitational instabilities that are needed to be removed. Most prominent dark energy proposals are based on cosmological constant $\Lambda$ [18, 19], quintessence [20, 21], k-essence [22, 23], phantom energy [24], quintom model [25, 26], geometric dark energy [27], holographic dark energy [28] and tachyons [29, 30], to name a few. It has been pointed out that quantum effects can yield a super-accelerated phase of cosmic expansion (without any need of introducing ghosts, phantoms or tachyons) and that these quantum effects yield stable solutions [31, 32, 33]. The precise determination of $\omega_{d e}$ is a more challenging and interesting problem in itself. Recent observational data gives the estimate $-1.67<\omega_{d e}<-1.05$ at $95 \%$ confidence level [34]. It also supports the notion of an evolving $\omega_{d e}$, hence it requires the parametric form $\omega_{d e}(z)$, where $z$ is the redshift parameter [35, 36, 37, 38, 39, 40]. For larger redsifts (quintessence dominated), $\omega_{d e}>-1$ while at some instant $\omega_{d e}=-1$ ( $\Lambda$ dominated era) and later it is $\omega_{d e}<-1$ (phantom regime). Therefore the CCP is rephrased as 'why now $\omega_{d e}=-1$ ?'

In the last few years, the CCP is addressed by invoking a non-minimal interaction between dark energy and dark matter (or simply 'matter' for convenience), the so-called interacting dark energy model [41, 42, 43, 44, 45, 46, 47, 48, 49, 50, 51, 52, 53]. The model involves a coupling parameter and an energy exchange term to govern the interaction. The energy exchange term is adjusted so as to satisfy the global conservation law for the interacting system. Moreover the interaction is dynamic i.e. the energy is exchanged between the interacting components with equal degree of freedom. It is exactly this feature that helps in maintaining the equilibrium of densities of the interacting components i.e. the ratio of energy densities roams around to unity. It is recently shown that the coupling parameter cannot be negative in order to avoid possible violation of the cherished second law of thermodynamics, however, small positive values are permissible to account the 
decay of dark energy into matter [54, 55, 56]. The notion of decay of dark energy into matter particles is well motivated from the theoretical arguments and henceforth predicts a matter dominated universe. Hence a universe governed by the interacting components can undergo deceleration phase preceded by an acceleration phase. Further, the interaction also saves the universe from undergoing an imminent 'big rip' (tearing apart of spacetime structure leading to a future spacelike singularity). This model also favors a bouncing universe (free from cosmological singularities like big bang, big rip, big crunch etc) since the model forces the components to interact, thereby avoiding cosmological over-densities.

We here extend our earlier work on interacting dark energy [57, 58] in the context of five dimensional brane-world model [59]. Last few decades have seen a considerable advancement on the theories of extra dimensions and have revealed deep insights about the structure of spacetime, elementary particles and forces of nature [60]. It has been suggested that the mysterious dark energy is also a manifestation of extra spatial dimensions 61]. We investigate the behavior of dark energy in the brane world model and determine various EoSs for the dark energy for different choices of the time dependent cosmological constant. All the effective EoS describe the phantom crossing scenario under certain conditions. It is also discussed that this generalized interacting dark energy model fairly resolves the CCP.

\section{Interacting dark energy model}

We start by assuming the background to be spatially homogeneous and isotropic FriedmannRobertson-Walker (FRW) spacetime, in the context of brane-world gravitation model, which is given by [62, 59]

$$
d s^{2}=d t^{2}-a^{2}(t)\left[\frac{d r^{2}}{1-k r^{2}}+r^{2}\left(d \theta^{2}+\sin ^{2} \theta d \phi^{2}\right)\right]-\zeta^{2}(t) d \psi^{2} .
$$

Here $k$ is a curvature parameter which refers to a spatially spherical $(k=+1)$, Minkowskian $(k=0)$ or hyperbolic $(k=-1)$ spacetime. Note that these cosmological models are also correspond to closed, flat or open respectively. Also $a(t)$ is the dimensionless scale factor while $\zeta$ is an arbitrary function of time $t$ and we choose it $\zeta(t)=a^{n}$, whereas $n$ is a constant. Moreover $\psi$ is the fourth spatial dimension. The spacetime is further assumed to contain two fluids namely matter and dark energy. The corresponding energy densities are $\rho_{m}$ and $\rho_{d e}$ while the respective pressures are $p_{m}=0$ (pressureless dust) and $p_{d e}=\omega_{d e} \rho_{d e} \neq 0$. The combined matter energy distribution is given by a perfect fluid stress energy tensor

$$
T_{\mu \nu}=\left(\rho_{m}+\rho_{d e}+p_{d e}\right) u_{\mu} u_{\nu}-p_{d e} g_{\mu \nu} .
$$


Here $u_{\mu}$ is the five-velocity vector which satisfies $u_{\mu} u^{\nu}=1$ with $\mu, \nu=0,1,2,3,4$. The first FRW equation is

$$
(n+1) H^{2}+\frac{k}{a^{2}}=\frac{1}{3 M_{p}^{2}}\left(\rho_{d e}+\rho_{m}\right)+\frac{\Lambda(t)}{3} .
$$

Here $M_{p}^{2}=(8 \pi G)^{-1}$ is the reduced Planck mass. The corresponding energy conservation equation is

$$
\dot{\rho}_{d e}+\dot{\rho}_{m}+(3+n)\left[\rho_{m}+\rho_{d e}\left(1+\omega_{d e}\right)\right] H=-M_{p}^{2} \dot{\Lambda},
$$

which can be decomposed into two non-conserving equations for both matter and dark energy as

$$
\begin{aligned}
\dot{\rho}_{d e}+(3+n)\left(1+\omega_{d e}\right) \rho_{d e} H & =-M_{p}^{2} \frac{\dot{\Lambda}}{2}-Q, \\
\dot{\rho}_{m}+(3+n) \rho_{m} H & =-M_{p}^{2} \frac{\dot{\Lambda}}{2}+Q .
\end{aligned}
$$

Note that addition of the above two equations leads to the energy conservation (4). Above $Q$ is the energy exchange term for the interaction. We here choose $Q=3 H b\left(\rho_{m}+\rho_{d e}\right)$ 63, 64, where $b$ is the coupling parameter (or transfer strength). Due to unknown nature of both dark energy and dark matter, the interaction term can not be derived from the first principles. It is worthy to note that if $Q<0$ than it will yield the energy density of dark energy to be negative at sufficiently early times, consequently the second law of thermodynamics can be violated [65] hence $Q$ must be positive and small. Because of the underlying interaction, the beginning of the accelerated expansion is shifted to higher redshifts.

Further the density parameters are

$$
\begin{aligned}
\Omega_{m} & =\frac{\rho_{m}}{\rho_{c r}}=\frac{\rho_{m}}{3 H^{2} M_{p}^{2}} \\
\Omega_{d e} & =\frac{\rho_{d e}}{\rho_{c r}}=\frac{\rho_{d e}}{3 H^{2} M_{p}^{2}} \\
\Omega_{k} & =\frac{k}{a^{2} H^{2}}
\end{aligned}
$$

Using the above parameters in Eq. (3), we obtain

$$
\Omega_{m}+\Omega_{d e}=1+n+\Omega_{k}-\frac{\Lambda(t)}{3 H^{2}} .
$$

We here define the dimensionless ratio of densities

$$
r_{x} \equiv \frac{\rho_{m}}{\rho_{d e}}=\frac{\Omega_{m}}{\Omega_{d e}} .
$$


To check how this density ratio evolves with time, we differentiate it w.r.t $t$ to get

$$
\dot{r}_{x}=\frac{d r_{x}}{d t}=\frac{\rho_{m}}{\rho_{d e}}\left[\frac{\dot{\rho}_{m}}{\rho_{m}}-\frac{\dot{\rho}_{d e}}{\rho_{d e}}\right] \equiv f\left(r_{x}\right) .
$$

Using Eqs. (5) and (6) in (12), we obtain

$$
\dot{r}_{x}=r_{x}(3+n) H\left[\omega_{d e}+\frac{\Gamma}{(3+n) H} \frac{1+r_{x}}{r_{x}}+\frac{\dot{\Lambda}\left(r_{x}-1\right)}{6 H^{3} \Omega_{m}(3+n)}\right],
$$

where

$$
\Gamma=3 H b\left(1+r_{x}\right)
$$

is the decay rate, related to $Q=\Gamma \rho_{d e}$. Thus as $r_{x}$ approaches 1 , the last term on right hand side in equation (13) becomes negligible. It is also termed as the 'soft coincidence' since $\left|\dot{r}_{x} / r_{x}\right| \leq H$ [66]. Further if $n=0$ then Eq. (13) reduces to the one discussed in [46]. Using Eq. (14) in (13), we obtain

$$
\dot{r}_{x}=r_{x}(3+n) H\left[\omega_{d e}+\frac{3 b\left(1+r_{x}\right)^{2}}{r_{x}(3+n)}+\frac{\dot{\Lambda}\left(r_{x}-1\right)}{6 H^{3} \Omega_{m}(3+n)}\right] .
$$

The critical points (or stationary solutions) are obtained by solving $\dot{r}_{x}=0$ to get

$$
r_{x}^{2}\left[\dot{\Lambda}+18 b H^{3} \Omega_{m}\right]+r_{x}\left[6 H^{3} \Omega_{m}\left\{6 b+\omega_{d e}(3+n)\right\}-\dot{\Lambda}\right]+18 b H^{3} \Omega_{m}=0 .
$$

The above equation yields two roots as

$$
\begin{aligned}
r_{x \pm}= & \frac{1}{2\left(\dot{\Lambda}+18 b H^{3} \Omega_{m}\right)}\left[\dot{\Lambda}-6 H^{3} \Omega_{m}\left\{6 b+\omega_{d e}(3+n)\right\}\right. \\
& \left. \pm \sqrt{-72 H^{3} \Omega_{m} b\left(\dot{\Lambda}+18 b H^{3} \Omega_{m}\right)+\left\{\dot{\Lambda}-6 H^{3}\left[6 b+(3+n) \omega_{d e}\right] \Omega_{m}\right\}^{2}}\right]
\end{aligned}
$$

It is recently shown in [50] that any model of interacting dark energy can resolve the cosmic coincidence problem if the function $f\left(r_{x}\right)$ satisfies

$$
\frac{d f}{d r}\left(r=r_{x i}\right)<0
$$

where $r_{x i}$ for $i=1,2, \ldots$ are the roots of $f\left(r_{x}\right)=0$. It needs to be stressed that not all roots will satisfy (18) but those which do satisfy it, are termed 'stable equilibrium points'. In our model, the condition (18) together with (17) yields

$$
f^{\prime}\left(r_{x \pm}\right)= \pm \frac{\sqrt{-72 H^{3} \Omega_{m} b\left(\dot{\Lambda}+18 b H^{3} \Omega_{m}\right)+\left[\dot{\Lambda}-6 H^{3}\left\{6 b+(3+n) \omega_{d e}\right\} \Omega_{m}\right]^{2}}}{6 H^{2} \Omega_{m}} .
$$


Hence it is clear that $f^{\prime}\left(r=r_{x-}\right)<0$ and $r_{x-}$ is the only stable equilibrium point of our model. Thus interacting dark energy model in brane-world gravitation theory fairly alleviates the CCP. Our next task is now to determine the effective EoS for dark energy.

The parameter $r_{x}$ in (11) is related to the density parameters (7) - (9) as

$$
r_{x}=\frac{1}{\Omega_{d e}}\left[1+n+\Omega_{k}-\Omega_{d e}-\frac{\Lambda(t)}{3 H^{2}}\right] .
$$

We further define the effective equations of state for dark energy and matter as [46]

$$
\omega_{d e}^{e f f}=\omega_{d e}+\frac{\Gamma}{3 H}, \quad \omega_{m}^{e f f}=-\frac{1}{r_{x}} \frac{\Gamma}{3 H},
$$

From Eq. (5) we have

$$
\omega_{d e}=-1-\frac{1}{(3+n) H \rho_{d e}}\left[Q+\dot{\rho}_{d e}+M_{p}^{2} \frac{\dot{\Lambda}}{2}\right] .
$$

Using Eq. (22) in (21), we get

$$
\omega_{d e}^{e f f}=-1-\frac{1}{(3+n) H \rho_{d e}}\left[\dot{\rho}_{d e}+M_{p}^{2} \frac{\dot{\Lambda}}{2}\right]+\frac{n \Gamma}{3(n+3) H},
$$

or we can write

$$
\omega_{d e}^{e f f}=-1-\frac{\dot{\rho}_{d e}}{(3+n) H \rho_{d e}}-\frac{\dot{\Lambda}}{6(3+n) H^{3} \Omega_{d e}}+\frac{n b\left(1+r_{x}\right)}{n+3} .
$$

We represent the dark energy by the modified Chaplygin gas (MCG) equation of state

$$
p_{d e}=A \rho_{d e}-\frac{B}{\rho_{d e}^{\alpha}},
$$

where $A, B$ and $\alpha$ are constant parameters. The MCG best fits with the 3-year WMAP and the SDSS data with the choice of parameters $A=-0.085$ and $\alpha=1.724$ [67] which are improved constraints than the previous ones $-0.35<A<0.025$ [68]. Recently it is shown that the dynamical attractor for the MCG exists at $\omega_{d e}=-1$, hence MCG crosses this value from either side $\omega_{d e}>-1$ or $\omega_{d e}<-1$, independent to the choice of model parameters [69]. A generalization of MCG is suggested in [70] by considering $B \equiv B(a)=B_{o} a^{\sigma}$, where $\sigma$ and $B_{o}$ are constants. The MCG is the generalization of generalized Chaplygin gas $p_{d e}=-B / \rho_{d e}^{\alpha}$ [71, 72] with the addition of a barotropic term. This special form also appears to be consistent with the WMAP 5-year data and henceforth the support the unified model with dark energy and matter based on generalized Chaplygin gas $[73,74]$. In the cosmological context, the Chaplygin gas was first 
suggested as an alternative to quintessence and demonstrated an increasing $\Lambda$ behavior for the evolution of the universe [75]. Recent supernovae data also favors the two-fluid cosmological model with Chaplygin gas and matter [76]. The density evolution of MCG is given by

$$
\rho_{d e}=\left(X+C_{1} a^{Y}\right)^{\frac{1}{1+\alpha}}
$$

where $X \equiv B /(1+A), Y \equiv 3(1+\alpha)(1+A)$ and $C_{1}$ is the constant of integration. The time derivative of $\rho_{d e}$ is given by

$$
\dot{\rho}_{d e}=-3(1+A) C_{1} H\left(X+C_{1} a^{Y}\right)^{\frac{-\alpha}{1+\alpha}} a^{Y} .
$$

Using Eqs. (24) to (27), we obtain the effective EoS for dark energy as

$$
\omega_{d e}^{e f f}=-1+\frac{3(1+A)}{3+n}\left(1-\frac{X}{\rho_{d e}^{1+\alpha}}\right)-\frac{\dot{\Lambda}}{6(3+n) H^{3} \Omega_{d e}}+\frac{n b\left(1+r_{x}\right)}{n+3} .
$$

Models with variable $\Lambda(t)$ are physically more appealing and theoretically rich in predictions as compared to constant $\Lambda$. If $\dot{\Lambda}<0$, it gives a decreasing behavior of $\Lambda$ with time. Physically it may explain inflationary expansion at earlier times while an accelerated expansion in current time. Similarly, $\dot{\Lambda}>0$ represents an increasing $\Lambda$ with time. It can be best interpreted in a model of bouncing cosmology where a universe is free from any potential cosmological singularities like the big bang one and bounces back near the imminent singularity. In such a scenario, a smaller $\Lambda$ corresponds to a deceleration phase followed by a smoothly evolving larger $\Lambda$ which results in a de Sitter like expansion. If we assume that this later expansion is driven by an exotic phantom energy $\left(\omega_{d e}<-1\right)$, then the later one decays into matter particles creating a matter dominated universe again [77]. Thus in a bouncing universe, an otherwise big rip singularity is replaced by the matter creation scenario. Hence if $\omega_{d e}<-1$, it eventually leads to two interesting results: first, the existence of a bouncing universe and second, the decay of dark energy into matter or the model of interacting dark energy [78, 45]. It is recently suggested using inhomogeneous EoS for dark energy that the dark energy dilution becomes faster in de Sitter expansion which involves strong interaction between dark energy and matter [79]. We shall now proceed to determine $\omega_{d e}^{e f f}$ by assuming dependencies of $\Lambda$ on various cosmological parameters and determine conditions under which it will become super-negative.

From Eq. (28) we see that $\omega_{d e}^{\text {eff }}<-1$ if $\dot{\Lambda}>0$ and $n>-3$. The scenario of dark energy dilution into matter arises for $b>0$ or $b \rightarrow 1$ if we restrict $0 \leq b \leq 1$ while a $b=0$ corresponds to a non-interacting dark energy model. It implies that for some specific values like $n=-1,-2$ the last term in Eq. (28) will also be negative. The case for $b<0$ refers to matter decay into dark energy, which is not relevant here. Let us choose $\Lambda(t)=C_{2} t^{\beta}$ than Eq. (28) yields

$$
\omega_{d e}^{e f f}=-1+\frac{3(1+A)}{3+n}\left(1-\frac{X}{\rho_{d e}^{1+\alpha}}\right)-\frac{C_{2} \beta t^{\beta-1}}{6(3+n) H^{3} \Omega_{d e}}+\frac{n b\left(1+r_{x}\right)}{n+3} .
$$


Next we choose $\Lambda(t)=C_{3} e^{\gamma t}$ which gives

$$
\omega_{d e}^{e f f}=-1+\frac{3(1+A)}{3+n}\left(1-\frac{X}{\rho_{d e}^{1+\alpha}}\right)-\frac{C_{3} \gamma e^{\gamma t}}{6(3+n) H^{3} \Omega_{d e}}+\frac{n b\left(1+r_{x}\right)}{n+3} .
$$

Thus in the above two Eqs. (29) and (30), $\dot{\Lambda}>0$ translates into $\beta>1$ and $\gamma>0$. Next we take $\Lambda(t)=C_{4} a^{\delta}$ which enables us to write

$$
\omega_{d e}^{e f f}=-1+\frac{3(1+A)}{3+n}\left(1-\frac{X}{\rho_{d e}^{1+\alpha}}\right)-\frac{C_{4} \delta a^{\delta}}{6(3+n) H^{2} \Omega_{d e}}+\frac{n b\left(1+r_{x}\right)}{n+3} .
$$

which can alternatively be written as

$$
\omega_{d e}^{e f f}=-1+\frac{3(1+A)}{3+n}\left(1-\frac{X}{\rho_{d e}^{1+\alpha}}\right)-\frac{C_{4} \delta\left[\frac{1}{C_{1}}\left(\rho_{d e}^{1+\alpha}-X\right)\right]^{\delta / Y}}{6(3+n) H^{2} \Omega_{d e}}+\frac{n b\left(1+r_{x}\right)}{n+3} .
$$

Here we require $\delta>0$ and $\delta>Y$ to get a super-negative EoS. If we take $\Lambda(t)=C_{5} H^{v}$ then we have

$$
\omega_{d e}^{e f f}=-1+\frac{3(1+A)}{3+n}\left(1-\frac{X}{\rho_{d e}^{1+\alpha}}\right)-\frac{C_{5} v H^{v-4} \dot{H}}{6(3+n) \Omega_{d e}}+\frac{n b\left(1+r_{x}\right)}{n+3} .
$$

The phantom crossing scenario is more prominently observed from $\dot{H}>0\left(\omega_{d e}<-1\right)$, $\dot{H}=0\left(\omega_{d e}=-1\right)$ and $\dot{H}<0\left(\omega_{d e}>-1\right)$. More specifically, at the transition $\omega_{d e}=$ $\omega_{d e}^{e f f}=-1$, we require

$$
b=-\frac{3(1+A)}{n\left(1+r_{x}\right)}\left(1-\frac{X}{\rho_{d e}^{1+\alpha}}\right) .
$$

Since $0 \leq b \leq 1$ and $n>-3$ for $\dot{\Lambda}>0$, we obtain a restriction $-3<n<0$ from Eq. (34). Lastly we take $\Lambda(t)=C_{6} \rho_{d e}^{\epsilon}$ hence we get

$$
\omega_{d e}^{e f f}=-1+\frac{3(1+A)}{3+n}\left(1-\frac{X}{\rho_{d e}^{1+\alpha}}\right)-\frac{C_{6} \epsilon \rho_{d e}^{\epsilon-1} \dot{\rho}_{d e}}{6(3+n) H^{3} \Omega_{d e}}+\frac{n b\left(1+r_{x}\right)}{n+3} .
$$

which we can be simplified to yield

$$
\omega_{d e}^{e f f}=-1+\frac{1+A}{3+n}\left(3+\frac{\epsilon C_{6} \rho_{d e}^{\epsilon}}{2 H^{2} \Omega_{d e}}\right)\left(1-\frac{X}{\rho_{d e}^{1+\alpha}}\right)+\frac{n b\left(1+r_{x}\right)}{n+3} .
$$

At the epoch of phantom crossing, we require $\omega_{d e}=-1=\omega_{d e}^{e f f}$ to get

$$
b=-\frac{1+A}{n\left(1+r_{x}\right)}\left(3+\frac{\epsilon C_{6} \rho_{d e}^{\epsilon}}{2 H^{2} \Omega_{d e}}\right)\left(1-\frac{X}{\rho_{d e}^{1+\alpha}}\right)
$$

Note that Eq. (37) is reduced to (34) if $\epsilon=0$. 


\section{Conclusion and discussion}

In the present work we attempted to resolve the cosmic coincidence problem in the context of five-dimensional brane world gravitation theory. The CCP is fairly alleviated since stable stationary solution exists for the dynamical system. The dark energy is represented by the modified Chaplygin gas and is further assumed to interact with the matter. This interaction leads to a phantom crossing scenario. We have also determined various effective EoS for dark energy using different choices of $\Lambda(t)$, since EoS of dark energy will change if it interacts with matter. This paper also presents a generalization of the work in [80] where it is shown that the dark energy with the MCG EoS crosses the phantom divide in the background of four dimensional FRW spacetime.

Models of interacting dark energy have taken considerable interest in recent years. The model mimics $\Lambda \mathrm{CDM}$ at early times while it gives a finite dark energy-dark matter ratio at late times so that coincidence problem is alleviated. It turns out that dark energy domination is merely a transient event and will be replaced by the dark matter dominant era once again [81]. It is recently shown that this model can resolve the cosmic age problem as well since simple dark energy cannot remove the problem [82]. The cosmic age predicted by the interacting model is predicted to be greater than the $\Lambda$ CDM model which is consistent with the observations and hence alleviates the cosmic age problem. Further, this model is also investigated in the context of loop quantum cosmology, a theory in which all cosmological singularities are avoided due to quantum effects [83]. It is also of some interest that the coupling parameter for the interaction yields a variable Newton's gravitational constant [84]. From the observational point of view, a small but non-vanishing interaction is reported from the analysis of the dynamics of 33 relaxed galaxy clusters like Abell A586 [85].

\section{Acknowledgment}

I am grateful to M.R. Setare, D. Polarski, G.S. Khadekar and V.K. Onemli for useful comments on this work.

\section{References}

[1] S. Perlmutter et al, Phys. Rev. Lett. 83 (1999) 670.

[2] S. Perlmutter et al, Ap. J. 517 (1999) 565.

[3] Y. Wang and M. Tegmark, Phys. Rev. Lett. 92 (2004) 241302

[4] A.G. Riess et al, Ap. J. 560 (2001) 49. 
[5] A.G. Riess et al, Astron. J. 116 (1998) 1009.

[6] D.N. Spergel et al, Ap. J. Suppl. 148 (2003) 175.

[7] J.L. Tonry et al, Ap. J. 594 (2003) 1.

[8] Y. Gong and C-K. Duan, Mon. Not. R. Astron. Soc. 352 (2004) 847.

[9] R.R. Caldwell et al, Phys. Rev. D 73 (2006) 023513.

[10] B. Ratra and P.J.E. Peebles, Phys. Rev. D 37 (1988) 3406.

[11] V. Sahni and A. Starobinsky, Int. J. Mod. Phys. D 15 (2006) 2105.

[12] S. Weinberg, Rev. Mod. Phys. 61 (1989) 1.

[13] E. Copeland, arXiv: 0603057 [hep-th].

[14] T. Padmanabhan, Phys. Rep. 380 (2003) 235.

[15] N. Arkani-Hamed et al, Phys. Rev. Lett. 85 (2000) 4434.

[16] M. Jamil and F. Rahaman, arXiv: 0810.1444 [gr-qc].

[17] S. Carroll et al, Phys. Rev. D 68 (2003) 023509.

[18] P.J. Steinhardt and N. Turok, Science 312 (2006) 1180.

[19] M. Ishak, Found. Phys. 37 (2007) 1470.

[20] Q-Guo Huang, arXiv: 0708.2760 [astro-ph].

[21] B. Ratra and P.J.E. Peebles, Ap. J. Lett. 325 (1998) 117.

[22] R. Das et al, arXiv: 0609014 [gr-qc]

[23] C. Armendariz-Picon et al, arXiv: 0006373 [astro-ph].

[24] R.R. Caldwell et al, Phys. Rev. Lett. 91 (2003) 071301.

[25] Yi-Fu Cai et al, Phys. Lett. B 646 (2007) 141

[26] Yi-Fu et al, Phys. Lett. B 651 (2007) 1

[27] E.V. Linder, New. Astron. Rev. 49 (2005) 93.

[28] M.R. Setare, Eur. Phys. J. C 50 (2007) 991.

[29] T. Padmanabhan, Phys. Rev. D 66 (2002) 021301.

[30] P.F. Gonzalez-Diaz, arXiv: 0408225 [hep-th].

[31] V.K. Onemli and R.P. Woodard, Class. Quant. Grav. 19 (2002) 4607.

[32] V.K. Onemli and R.P. Woodard, Phys. Rev. D 70 (2004) 107301.

[33] T. Brunier et al, Class. Quant. Grav. 22 (2005) 59.

[34] A. De La Macorra et al, Astropart. Phys. 27 (2007) 406.

[35] M. Chevallier and D. Polarski, Int. J. Mod. Phys D 10 (2001) 213.

[36] E.M. Barboza and J.S. Alcaniz, Phys. Lett. B 666 (2008) 415. 
[37] J. Weller and A. Albrecht, Phys. Rev. D 65 (2002) 103512.

[38] E.V. Linder, Phys. Rev. Lett. 90 (2003) 091301.

[39] G. Efstathiou, Mon. Not. R. Astron. Soc. 342 (2000) 801.

[40] A. Upadhye, Nuc. Phys. B (Proc. Suppl.) 173 (2007) 11.

[41] W.F. Wang and S.Z. Yang, Phys. Lett. B 668 (2008) 79.

[42] M. Szydlowski, Phys. Lett. B 632 (2006) 1.

[43] J.D. Barrow and T. Clifton, Phys. Rev. D 73 (2006) 103520

[44] A. de la Macorra, arXiv: astro-ph/0701635v1

[45] N.P. Neto and B.M.O. Fraga, Gen. Relativ. Gravit. 40 (2008) 1653.

[46] M.R. Setare, Eur. Phys. J. C 52 (2007) 689.

[47] S. Nojiri and S.D. Odintsov, arXiv: hep-th/0505215

[48] W. Zimdahl and D. Pavon, Gen. Relativ. Gravit. 36 (2004) 1483.

[49] L.P. Cimento et al, Phys. Rev. D 67 (2003) 087302.

[50] T. Gonzalez and I. Quiros, Class. Quantum Gravit. 25 (2008) 175019.

[51] T. Koivisto and D.F. Mota, arXiv: 0707.0279 [astro-ph]

[52] S. Das and N. Banerjee, Gen. Relativ. Gravit. 38 (2006) 785.

[53] N. Cruz et al, Phys. Lett. B 663 (2008) 338.

[54] C. Feng et al, Phys. Lett. B 665 (2008) 111.

[55] S. Li et al, arXiv: 0809.0617 [gr-qc].

[56] M. Jamil and M.A. Rashid, arXiv: 0802.1144 [astro-ph]

[57] M. Jamil and M.A. Rashid, Eur. Phys. J. C 58 (2008) 111.

[58] M. Jamil and M.A. Rashid, Eur. Phys. J. C 56 (2008) 429.

[59] O. Gron and S. Hervik, Einstein's General Theory of Relativity, Springer 2007

[60] J. Polchinski, String Theory I, II, Cambridge Univ. Press, 1998.

[61] M. Ito, arXiv:0812.4326 [gr-qc].

[62] G.S. Khadekar et al, Int. J. Theor. Phys. (2008) DOI 10.1007/s10773-008-9810-2

[63] Z-Kuan Guo and Y-Zhong Zhang, Phys. Rev. D 71 (2005) 023501.

[64] S. del Campo et al, arXiv:0812.2210v1 [gr-qc].

[65] J.S. Alcaniz and J.A.S. Lima, Phys. Rev. D 72 (2005) 063516.

[66] S. del Campo et al, Phys. Rev. D 74 (2006) 023501.

[67] J. Lu et al, Phys. Lett. B 662 (2008) 87.

[68] L. Dao-Jun and L. Xin-Zhou, Chin. Phys. Lett. 22 (2005) 1600. 
[69] H. Jing et al, Chin. Phys. Lett. 25 (2008) 347.

[70] U. Debnath, arXiv: 0710.1708 [gr-qc].

[71] T. Barreiro and A.A. Sen, Phys. Rev. D 70 (2004) 124013.

[72] D. Carturan and F. Finelli, Phys. Rev. D 68 (2003) 103501.

[73] T. Barreiro et al, Phys. Rev. D 78 (2008) 043530.

[74] M. Makler et al, Phys. Lett. B 555 (2003) 1.

[75] A. Kamenshchik et al, Phys. Lett. B 511 (2001) 265.

[76] G. Panotopoulos, Phys. Rev. D 77 (2008) 107303.

[77] R. Curbelo et al, arXiv: 0502141v5 [astro-ph].

[78] A. Baushev, arXiv: 0809.0235 [astro-ph].

[79] W. Chakraborty and U. Debnath, Phys. Lett. B (2008) doi:10.1016/j.physletb.2008.01.054

[80] Y.B. Wu et al, Gen. Relativ. Gravit. 39 (2007) 653.

[81] G. Caldera-Cabral et al, arXiv: 0812.1827 [gr-qc].

[82] S. Wang and Y. Zhang, arXiv: 0809.3627v2 [astro-ph].

[83] S. Chen et al, arXiv: 0808.3482v2 [gr-qc].

[84] F. P. Zen et al, arXiv: 0809.3847 [hep-th].

[85] O. Bertolami et al, Phys. Lett. B 654 (2007) 165. 